УДК 004.89

DOI: $10.15827 / 2311-6749.18 .1 .6$

\title{
ПОДХОДЫ К СОЗДАНИЮ КААЬКУАЯТОРА С РУКОПИСНЫМ ВВОДОМ
}

\author{
С.А. Беляев, к.т.н., доиент, bserge@bk.ru; Д.А. Аапиевич, студентка, \\ darya.laptsevich@gmail.com \\ (Санкт-Петербургский государственный электротехнический университет "АЭТИ" \\ им. В.И. Ульянова (Иенина) (СПбГЭТУ "АЭТИ»), ул. Профрессора Попова, 5, \\ г. Санкт-Петербург, 197376, Россия)
}

В статье рассмотрены подходы к созданию калькулятора с рукописным вводом, исследованы существующие решения, их достоинства и недостатки. На основе выявленных особенностей готовых решений предложены собственное решение, его математическая модель, архитектура и сценарии использования предлагаемого решения.

Приведены результаты экспериментов для различных методов классификации рукописного текста, а также для параметров, улучшающих работу классификатора с использованием образцов рукописного написания цифр MNIST.

Ключевые слова: калькулятор с рукописным вводом, многоклассовая классификация, метод опорных векторов, MNIST, распознавание текста.

Сегодня техника окружает нас всюду, более того, ее интеллектуальные способности развиваются год от года, поэтому для разработчиков ПО важно добиться удобства и практичности в «общении» пользователя с техническими средствами.

В числе самых популярных инструментов человеко-машинного интерфейса - голосовой ввод и ввод рукописного текста. Эти возможности стали нормой и функциональным инструментом для современного пользователя.

Практически каждый человек использует калькулятор. Любая сфера деятельности предполагает какие-либо расчеты: основной задачей бухгалтера является формирование баланса организации, домохозяйка подсчитывает стоимость коммунальных услуг, студент вычисляет ответ математической задачи. Каждый из нас сталкивается с вычислениями, которые в некоторых случаях затруднительно выполнять без вычислительной машины.

У людей различные привычки и уникальный опыт: одним удобен голосовой ввод, другие привыкли к клавишам калькулятора, а кому-то проще пользоваться рукописным вводом. В статье предлагается рассмотреть возможности по созданию калькулятора с рукописным вводом.

\section{Существующие решения}

В настоящее время существуют решения, предлагающие рукописный ввод формул, в некоторых случаях с последующим их вычислением:

- MyScript Calculator [1];

- Touch-Calculator [2];

- Mathematical Expression Recognition [3].

Практически универсальным на сегодняшний день является MyScript Calculator: он распознает математические выражения целиком, знает множество математических функций, общепринятых обозначений, распознает степени, индексы, дроби и даже умеет искать недостающие части уравнений. Существуют версии для Android и iOS.

Реализация Touch-Calculator с рукописным вводом осуществляется для системы MacOS. Калькулятор имеет как интерфейс с «кнопочными» цифрами и математическими знаками, так и поле для рукописного ввода. Распознавание символа проходит за доли секунды, а ошибка классификации очень близка к нулю. Единственный недостаток данной реализации - распознавание происходит только по одному символу.

Классификация символов математического выражения в Mathematical Expression Recognition происходит некачественно (из 100 введенных символов 21 был распознан некорректно, то есть ошибка классификации достигает $21 \%$ ). Приложение осуществляет распознавание математического выражения без определения результата вычислений. Классификация выражения длиной в 10 символов происходит за 5-7 секунд. Существующая реализация обладает нестилизованным интерфейсом.

В таблице 1 показано наличие реализаций существующих решений для различных платформ. В таблице 2 представлен сравнительный анализ функционала существующих решений. 
Наличие реализаций существующих решений для различных платформ

\begin{tabular}{|l|c|c|c|c|c|c|}
\hline \multicolumn{1}{|c|}{ Решение } & MacOS & Windows & Linux & Android & iOS & Web \\
\hline MyScript Calculator & Нет & Нет & Нет & Да & Да & Нет \\
\hline Touch-Calculator & Да & Нет & Нет & Нет & Нет & Нет \\
\hline $\begin{array}{l}\text { Mathematical Expression } \\
\text { Recognition }\end{array}$ & Нет & Нет & Нет & Нет & Нет & Да \\
\hline
\end{tabular}

Сравнительный анализ функционала существующих решений

\begin{tabular}{|l|c|c|c|}
\hline \multicolumn{1}{|c|}{ Решение } & $\begin{array}{c}\text { Точность } \\
\text { классификации, \% }\end{array}$ & $\begin{array}{c}\text { Распознавание } \\
\text { выражения целиком }\end{array}$ & $\begin{array}{c}\text { Вычисление } \\
\text { значения }\end{array}$ \\
\hline MyScript Calculator & $\approx 99$ & Да & Да \\
\hline Touch-Calculator & $\approx 98$ & Нет & Да \\
\hline $\begin{array}{l}\text { Mathematical Expression } \\
\text { Recognition }\end{array}$ & $\approx 79$ & Да & Нет \\
\hline
\end{tabular}

Существуют реализации для мобильных устройств, а также реализация для платформы MacOS. Caмым востребованным решением является web-версия приложения: ее можно использовать для любой из существующих операционных систем, как настольных, так и мобильных. У существующего wеbприложения рукописного калькулятора можно выделить несколько недостатков: низкое качество классификации ( $\approx 79 \%)$ долгое распознавание выражения (10 символов за 5 секунд) и он не производит вычисление введенного выражения. В результате исследования существующих решений предлагается рассмотреть альтернативную реализацию web-версии калькулятора с рукописным вводом, обеспечивающую распознавание математического выражение с минимальной ошибкой классификации и его вычисление.

Математическая модель решения

Решение задачи рукописного ввода для калькулятора осуществляется в несколько этапов:

- определение границ каждого из символов (сегментация изображения);

- определение класса каждого символа;

- посимвольный разбор получившейся строки и вычисление результата;

- обучение классификатора.

В качестве языка программирования для серверной части приложения выбран язык Руthon, так как он предоставляет множество готовых библиотек для работы с рукописным текстом, его классификацией и сегментацией.

Для определения границ символов и посимвольного разбора строки в Руthon существуют библиотеки, которые можно применить к серверной части приложения без изменений или предварительной обработки данных. Для определения класса каждого объекта существуют библиотеки с готовыми реализациями методов классификации, однако необходимо подбирать параметры для качественной работы методов.

Для выполнения классификации рассмотрим следующие методы:

- мультиномиальная логистическая регрессия;

- наивный Байесовский классификатор;

- метод $k$ ближайших соседей;

- метод Парзеновского окна;

- метод опорных векторов.

\section{Мультиномиальная логистическая регрессия}

Модель мультиномиальной логистической регрессии [4] предлагает гипотезу, которая оценивает вероятность $P=(y=k \mid x)$, где $k=1, \ldots, K$, то есть вероятность, с которой каждый объект исходного множества принадлежит каждому из $K$ классов.

Далее определяются параметры модели. Подбор параметров в данной модели осуществляется при помощи минимизации функции стоимости, которая описывается следующим образом:

$$
J(w)=-\left[\sum_{i=1}^{N} \sum_{k=1}^{K} 1\left\{y_{i}=k\right\}\left(\log \left(e^{\left(w_{k}^{T} x_{i}\right)}\right)-\log \left(\sum_{j=1}^{K} e^{w_{j}^{T} x_{i}}\right)\right)\right] .
$$

При использовании мультиномиальной регрессии вероятность попадания объекта в класс определяется по формуле 


$$
P\left(y_{i}=k \mid x_{i} ; w\right)=\frac{e^{w_{k}^{T} x_{i}}}{\sum_{j=1}^{K} e^{w_{j}^{T} x_{i}}} .
$$

Минимизация функции стоимости осуществляется с использованием градиента.

В результате получаем параметры математической модели, которые будут использованы для подтверждения гипотезы. С ее применением по параметрам входного тестового объекта определяется его класс.

\section{Наивный Байесовский классификатор}

В основе модели Байесовского классификатора $[5,6]$ лежит теорема Байеса:

$$
P(k \mid x)=\frac{P(x \mid k) P(k)}{P(x)},
$$

где $P(k \mid x)$ - вероятность того, что объект $x$ принадлежит классу $k ; P(x \mid k)$ - вероятность того, что объект $x$ встречается среди объектов класса $k ; P(k)$ - безусловная вероятность встретить объект класса $k, P(x)-$ безусловная вероятность объекта $x$.

Байесовский классификатор осуществляет классификацию с использованием оценки априорного максимума:

$$
k_{\max }=\arg \max _{k \in K} \frac{P(x \mid k) P(k)}{P(x)} .
$$

Вероятность $P(x)$ для всех объектов одинакова, формула может быть записана в следующем виде:

$$
k_{\text {max }}=\arg \max _{k \in K} P(x \mid k) P(k) \text {. }
$$

Для вычисления класса объекта рассчитывается вероятность, с которой объект принадлежит классу $k$.

Объект представляется в виде набора признаков, вероятности которых условно не зависят друг от друга:

$$
P(x \mid k)=P\left(w_{1} \mid k\right) P\left(w_{2} \mid k\right) \ldots P\left(w_{N} \mid k\right)=\prod_{i=1}^{N} P\left(w_{i} \mid k\right) .
$$

Наивный Байесовский классификатор приобретает вид:

$$
k_{\max }=\arg \max _{k \in K} P(k) \prod_{i=1}^{N} P\left(w_{i} \mid k\right) .
$$

При большом количестве признаков происходит многократное перемножение чисел меньше единицы, поэтому используется формула в логарифмическом пространстве:

$$
k_{\max }=\arg \max _{k \in K}\left(\log P(k)+\sum_{i=1}^{N} \log P\left(w_{i} \mid k\right) .\right.
$$
борке:

Безусловная вероятность того, что объект принадлежит классу $k$, оценивается по тренировочной вы-

$$
P(k)=N_{k} / N,
$$

где $N_{k}$ - количество объектов в тренировочной выборке, принадлежащих классу $k$; $N$ - количество всех объектов в тренировочной выборке.

Оценка параметров Байесовской модели:

$$
P\left(w_{i} \mid k\right)=\frac{w_{i k}+a}{a+\sum_{j=1}^{N} w_{j k}},
$$

где $w_{i k}$ - общее количество элементов с заданным значением признака $i$ в классе $k ; a$ - параметр сглаживания, значение которого всегда больше 0, вводится, чтобы значение вероятности не принимало нулевое значение.

Окончательный вариант наивного Байесовского классификатора принимает следующий вид:

$$
k_{\text {max }}=\arg \max _{k \in K} \log \frac{N_{k}}{N} \sum_{i=1}^{M} \log \frac{w_{i k}+a}{a+\sum_{j=1}^{M} w_{j k}} .
$$

Используя данную формулу, определяется класс объекта. Параметр а подбирается экспериментальным путем.

\section{Метод $\boldsymbol{k}$ ближайших соседей}

В модели метода $k$ ближайших соседей [7] выбирается количество ближайших соседей $k$, по которым будет происходить оценка классифицируемого объекта. 
Значение $k$ определяется по критерию скользящего контроля [8].

Все объекты тренировочной выборки располагаются в следующей последовательности:

$$
\rho\left(x, x_{i 1}\right) \leq \rho\left(x, x_{i 2}\right) \leq \ldots \leq \rho\left(x, x_{i N}\right),
$$

где $\rho\left(\mathrm{x}, x_{i n}\right)$ - функция расстояния.

Из полученной последовательности выбирается $k$ первых элементов, по которым будет определяться принадлежность классифицируемого объекта к какому-либо классу.

Функция для классификации объекта выглядит следующим образом:

$$
f(x)=\arg \max _{y \in Y} \sum_{i=1}^{k}\left(y\left(x_{x i}\right)=y\right) w_{x i},
$$

где $w_{i x}$ - вес $i$-го объекта из упорядоченной по расстоянию тренировочной выборки для объекта $x$.

Используя полученную функцию и подобранное количество ближайших соседей, определяется класс объекта.

\section{Метод Парзеновского окна}

В основе модели метода Парзеновского окна [9] лежит модель метода $k$ ближайших соседей. В методе ближайших соседей выбирается количество ближайших соседей $k$, по которым будет происходить оценка классифицируемого объекта. В данном алгоритме по подобной логике выбирается ширина Парзеновского окна.

Как и для параметра $k$ из метода ближайших соседей, значение $h$ определяется по критерию скользящего контроля [8].

Все объекты тренировочной выборки располагаются в последовательности, располагающейся по возрастанию расстояний до объектов:

где $\rho\left(\mathrm{x}, x_{i n}\right)-$ функция расстояния.

$$
\rho\left(x, x_{i 1}\right) \leq \rho\left(x, x_{i 2}\right) \leq \ldots \leq \rho\left(x, x_{i N}\right),
$$

Функция для классификации объекта выглядит следующим образом:

$$
f(x)=\arg \max _{y \in Y} \sum_{i=1}^{n}\left(y\left(x_{x i}\right)=y\right) K\left(\frac{1}{h} \rho\left(z, x_{x i}\right)\right),
$$

где $K-$ ядро.

Ядро может быть выбрано из следующего набора:

- ядро Епанечникова;

- квартическое;

- треугольное;

- гауссовское;

- прямоугольное.

Функция ядра подбирается экспериментальным путем. Используя полученную функцию и выбранную ширину окна, определяется класс объекта.

\section{Метод опорных векторов}

Модель метода опорных векторов [8] была разработана для бинарной классификации, однако существует и модификация для многоклассовой. Все объекты тренировочной выборки представлены в $k$-мерном пространстве в виде вектора размерности $k$. Для разделения имеющихся объектов в пространстве используется так называемая плоскость классификатора, которая представляет собой гиперплоскость размерностью $k-1$. Таких плоскостей можно провести бесконечно большое количество. В алгоритме опорных векторов лучшей разделяющей плоскостью считается плоскость, расстояние от которой до каждого из классов максимально. Пространство оказывается разделенным на участки, каждый из которых соответствует какому-либо классу.

После того, как плоскость проведена, определяется положение каждого классифицируемого объекта. Ему присваивается класс, который соответствует участку, в который попал классифицируемый объект.

Пусть $X$ - пространство объектов тренировочной выборки, $Y$ - пространство ответов. В случае, когда необходимо разделить линейно неразделимую выборку, все элементы этой выборки помещаются в пространство, размерность которого выше размерности заданной выборки, используется отображение $\varphi: R^{(n)} \rightarrow X$. Это отображение выбирается таким образом, чтобы в новом пространстве выборка была линейно-разделимой. $X$ является пространством со скалярным произведением. Разделяющая функция в данном случае имеет следующий вид:

$$
f(x)=\left(w, \varphi\left(x_{i}\right)\right)+b, w=\sum_{i=1}^{N} \lambda_{i} y_{i} \varphi\left(x_{i}\right),
$$


где коэффициенты $\lambda_{i}$ зависят от $y_{i}$ и от значения ядра. Ядром является любая функция вида $K(x, y)$, являющаяся симметричной и неотрицательно определенной.

В данной статье рассматривается ядро вида:

$$
K(x, y)=e^{-\gamma\|x-y\|^{2}}, \gamma>0 .
$$

Такое ядро называется радиальной базисной функцией (RBF).

\section{Архитектура}

Для разработки калькулятора с рукописным вводом предлагается архитектура, представленная на рисунке 1 .

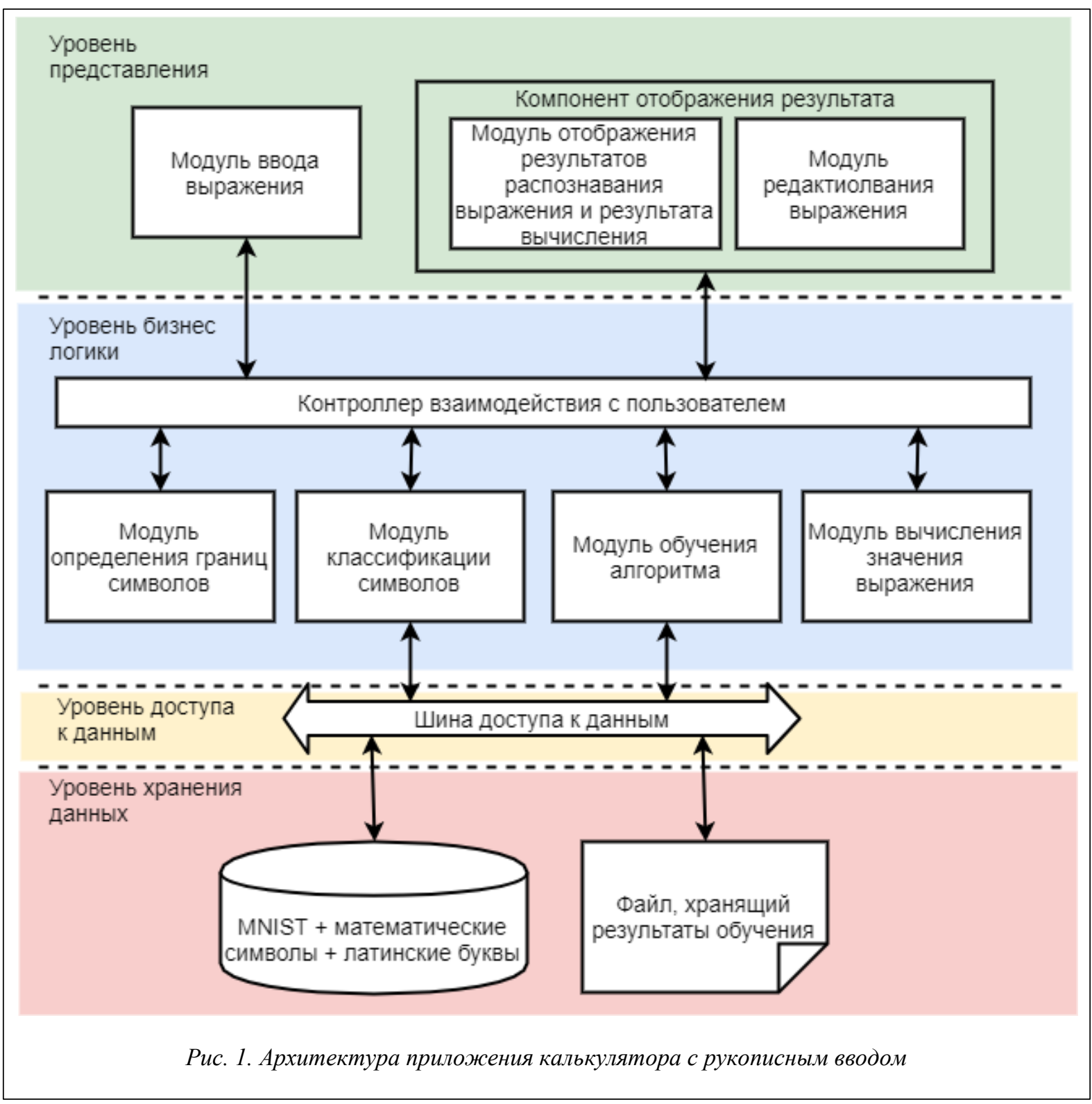

Уровень представления

На уровне представления считывается введенное пользователем изображение, отправляется запрос к серверной части приложения для распознавания или редактирования текста, отображаются результат распознавания выражения и его результат.

Модуль ввода выражения позволяет пользователю написать математическое выражение, считать его и передать его серверной части приложения.

Компонент отображения результата отвечает за работу модуля отображения результата распознавания выражения и результата вычислений, а также модуля редактирования выражения.

Модуль отображения результата распознавания выражения и результата вычислений позволяет отобразить на интерфейсе результат распознавания и классификации рукописного текста, а также его резуль- 
тат. Модуль производит взаимодействие с серверной частью приложения, отвечающей за классификацию рукописных символов и их редактирование.

Модуль редактирования выражения позволяет пользователю редактировать некорректно распознанные символы рукописного математического выражения, передает исправленный вариант серверной части приложения, отвечающей за вычисление результата.

Уровень бизнес-логики

На уровне бизнес-логики происходят принятие запроса от пользователя на распознавание изображения или на редактирование выражения, сегментация полученного изображения, классификация символов, вычисление результата выражения, обучение классификатора, формирование ответа пользователю с распознанным математическим выражением и результатом его вычисления. Также на данном уровне формируются запросы на получение данных из БД.

Контроллер взаимодействия с пользователем принимает запросы от пользователей, перенаправляет их на конкретные модули серверной части приложения, отправляет ответы сервера на запросы клиентов.

Модуль определения границ и символов отвечает за сегментацию полученного выражения, определяет границы символов и отправляет результат на контроллер.

Модуль классификации символов принимает на вход изображения отдельных символов, запрашивает данные из файла, в котором содержатся результаты обучения классификатора, при помощи полученных параметров производит классификацию каждого из полученных изображений, отправляет результат контроллеру.

Модуль вычисления значения выражения принимает на вход набор символов в определенном порядке, распознает правильный порядок вычислений и производит их, затем возвращает результат на контроллер.

Модуль обучения алгоритма используется только в тех случаях, когда БД символов для обучения была изменена или дополнена. Модуль принимает запрос на обучение; формирует запрос в БД символов; получает данные из БД; используя полученные данные, обеспечивает обучение алгоритма; записывает результат в файл; отправляет уведомление контроллеру о том, что обучение окончено.

Уровень доступа к даннылм

На данном уровне обеспечивается работа шины работы с данными, которая является контроллером запросов к БД и ответов на них.

Уровень хранения данных

Содержит БД для обучения классификатора и сериализованный объект обученного классификатора.

Сценарии использования

Рассмотрим возможные сценарии использования приложения.

1. Распознавание пользователем выражения:

- заходит в приложение;

- видит поле для ввода выражения, кнопку «Вычислить значение выражения», кнопку «Очистить поле для ввода»;

- вводит математическое выражение в поле для ввода рукописного текста;

- нажимает кнопку «Вычислить значение выражения»;

- видит под кнопками текстовое поле с полученным после распознавания выражением и его результат.

2. Редактирование пользователем результата распознавания:

- заходит в приложение;

- видит поле для ввода выражения, кнопку «Вычислить значение выражения», кнопку «Очистить поле для ввода»;

- вводит математическое выражение в поле для ввода рукописного текста;

- нажимает кнопку «Вычислить значение выражения»;

- видит под кнопками текстовое поле с полученным после распознавания выражением и его результат;

- получает некорректное значение;

- наводит курсор на распознанное выражение и ставит его на место, где необходимо произвести редактирование;

- редактирует выражение и автоматически видит новый ответ.

3. Повторное распознавание выражения:

- пользователь находится в приложении;

- видит поле для ввода выражения с введенным на нем выражением, кнопку «Вычислить значение выражения», кнопку «Очистить поле для ввода», распознанное предыдущее выражение и его результат;

- нажимает кнопку «Очистить поле для ввода» и видит чистое поле для ввода;

- вводит математическое выражение в поле для ввода рукописного текста;

- нажимает кнопку «Вычислить значение выражения»; 
- видит под кнопками текстовое поле с полученным после распознавания выражением и его результат.

Схема работы приложения, построенного на основании предложенной архитектуры и описанных сценариев использования, приведена на рисунке 2.

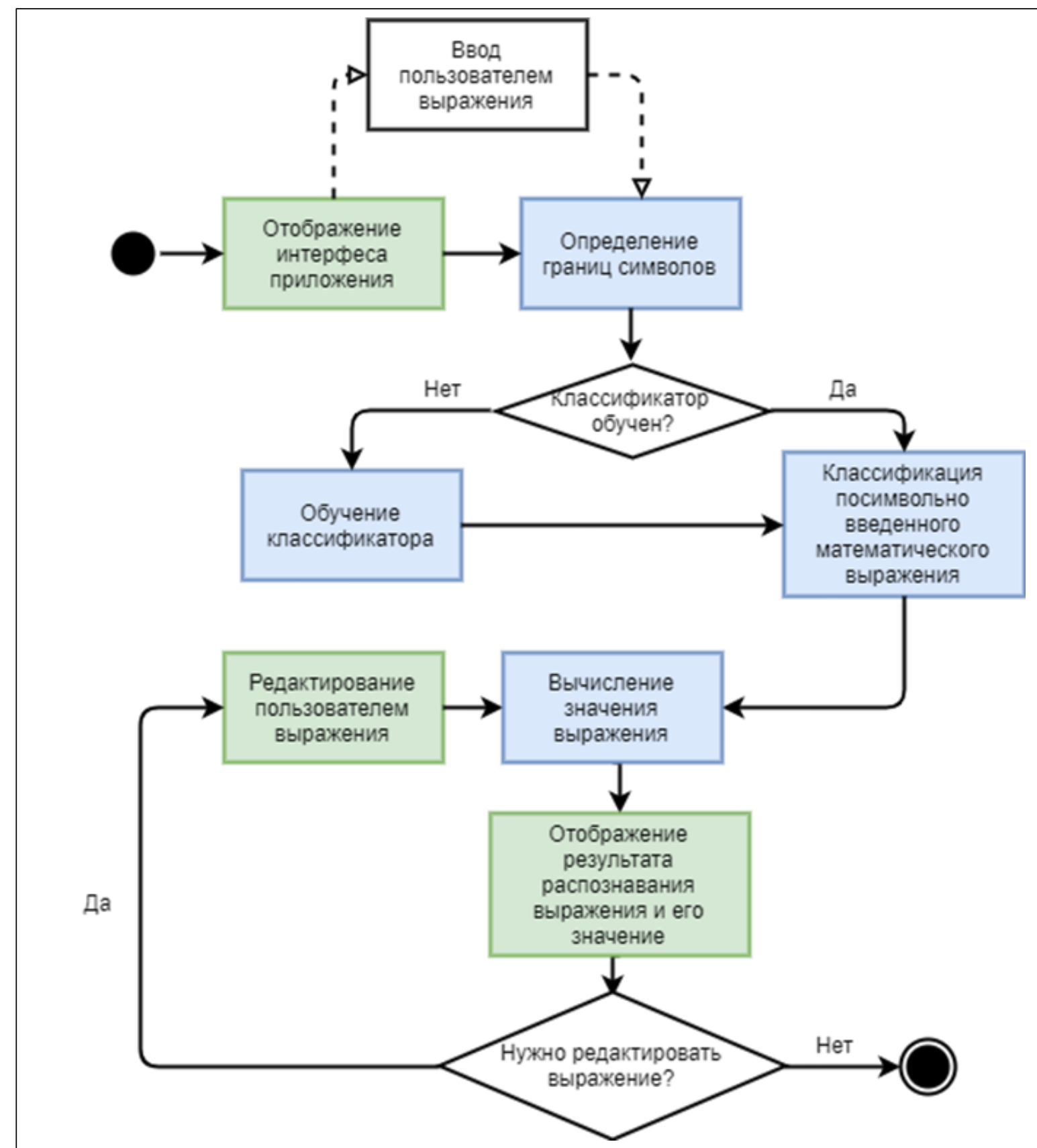

Рис. 2. Работа приложения с использованием предложенной архитектуры и сценария использования

\section{Полученные результаты}

Классификатор нужно обучить таким образом, чтобы он осуществлял классификацию с максимальной точностью. Исследуем каждый из рассмотренных методов классификации, используя в качестве обучающей выборки БД MNIST.

Мультиномиальная логистическая регрессия.

Данный метод зависит от параметра $\lambda$, который используется в функции стоимости.

Результаты исследования зависимости точности классификации от выбора параметра $\lambda$ представлены на рисунке 3. 


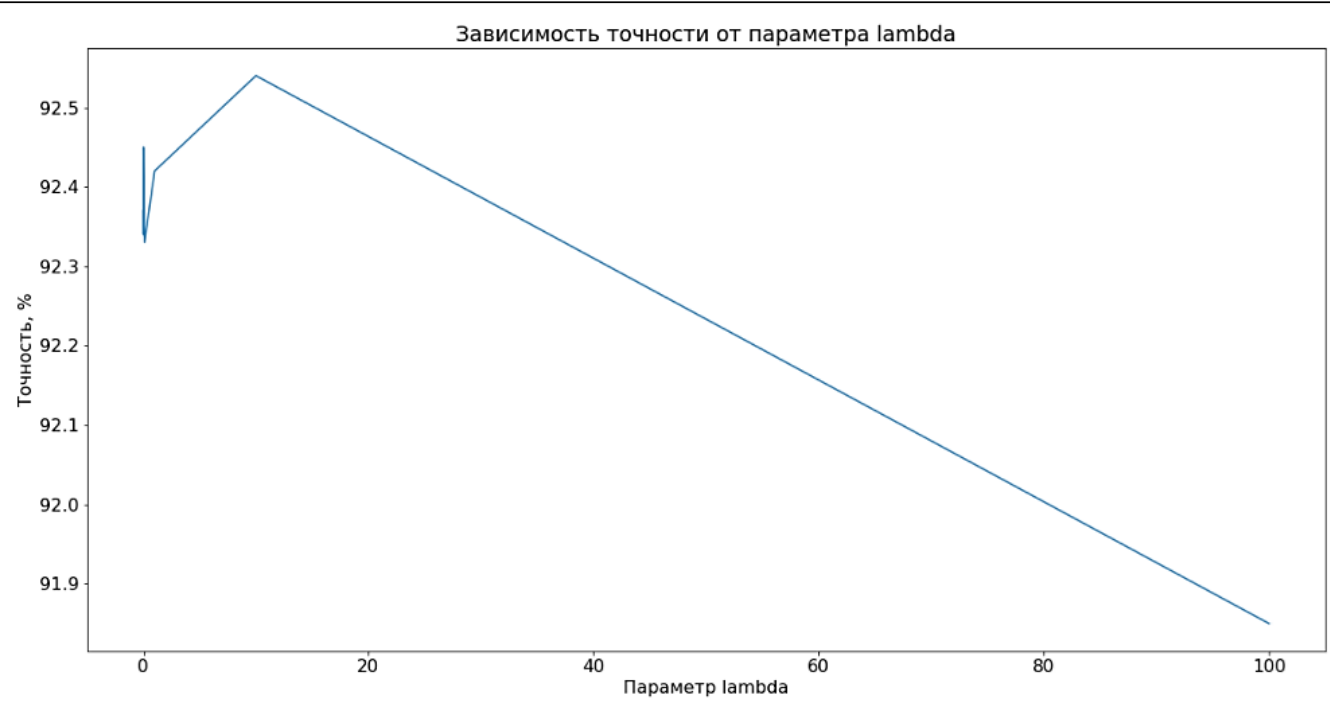

Рис. 3. Зависимость точности классификации методом мультиномиальной регрессии от выбора параметра $\lambda$

Достигнута точность 92,54 \% при значении параметра $\lambda=0.01$.

Наивный Байесовский классификатор.

Данный классификатор зависит от параметра сглаживания, благодаря которому значение функции классификатора не содержит 0 в знаменателе.

Результаты исследования зависимости точности классификации от параметра сглаживания представлены на рисунке 4 .

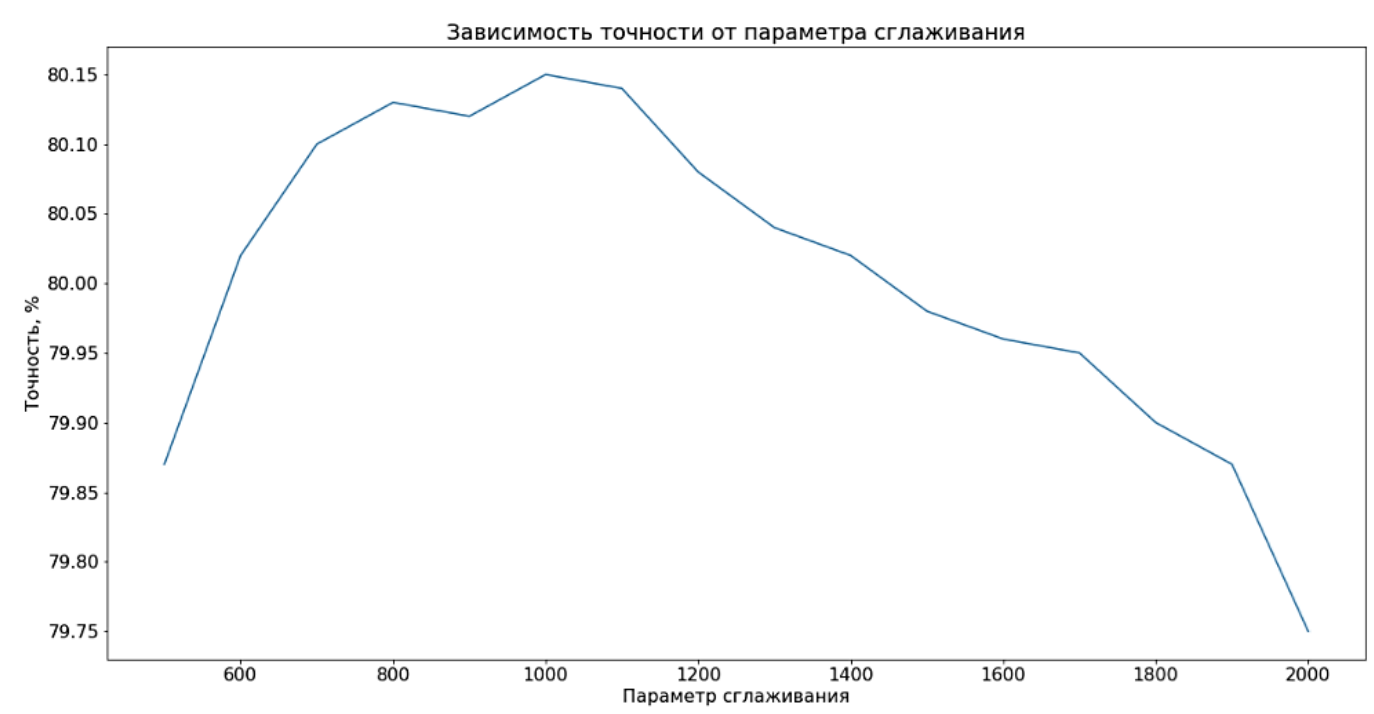

Рис. 4. Зависимость точности классификачии методом наивного Байесовского классификатора от параметра сглаживания

Достигнута точность 80,15 \% при значении параметра сглаживания, равном 1000.

Метод к ближайших соседей.

Данный классификатор зависит от количества соседей, по которым происходит классификация объекта.

Результаты исследования зависимости точности классификации от количества ближайших соседей представлены на рисунке 5. 


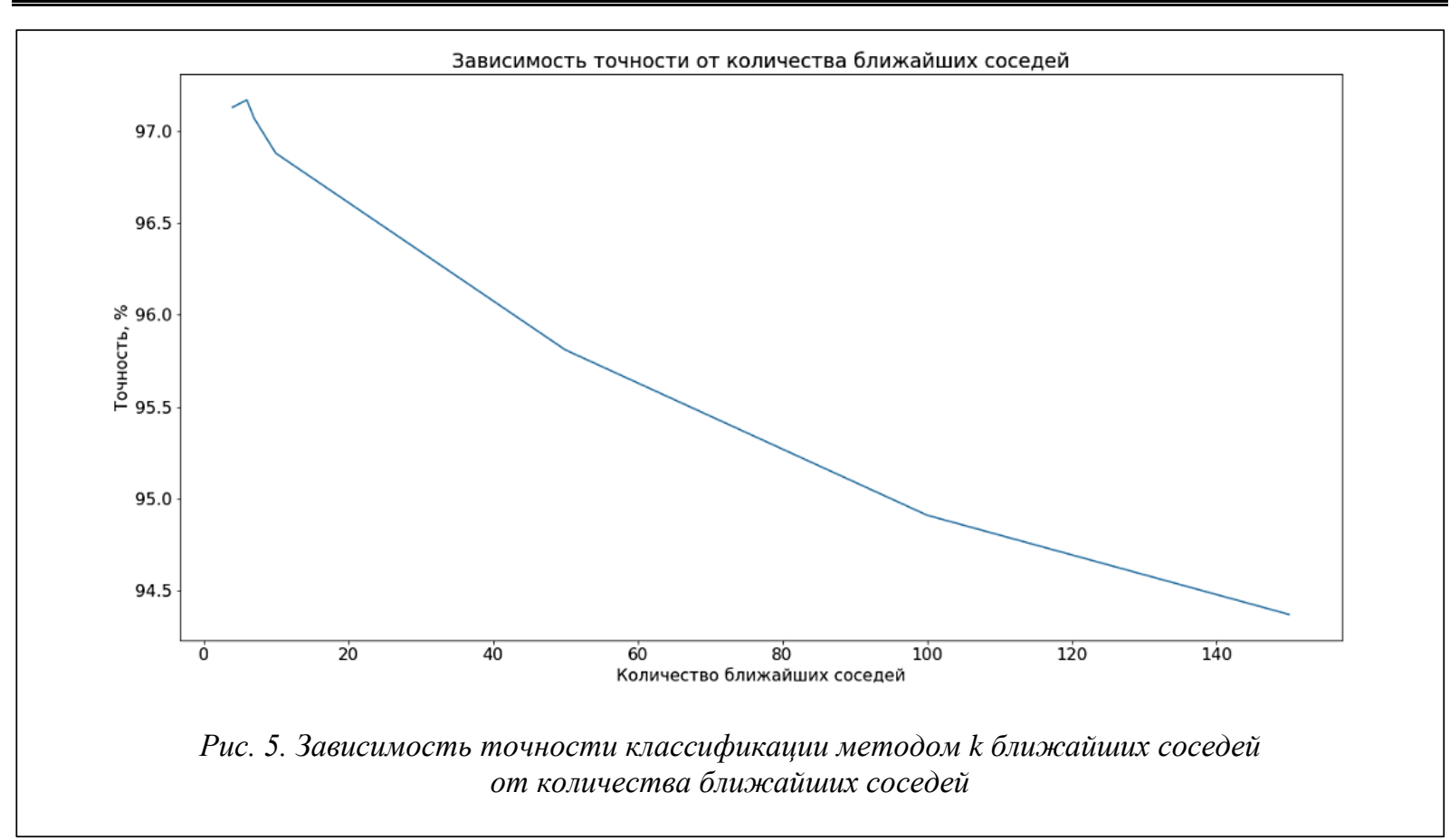

Достигнута точность 97,17 \% при количестве ближайших соседей равном 6.

Метод Парзеновского окна.

Данный классификатор зависит от ширины выбранного окна и от выбранного ядра.

Результаты исследования зависимости точности классификации от ширины окна и от вида ядра представлены на рисунке 6.

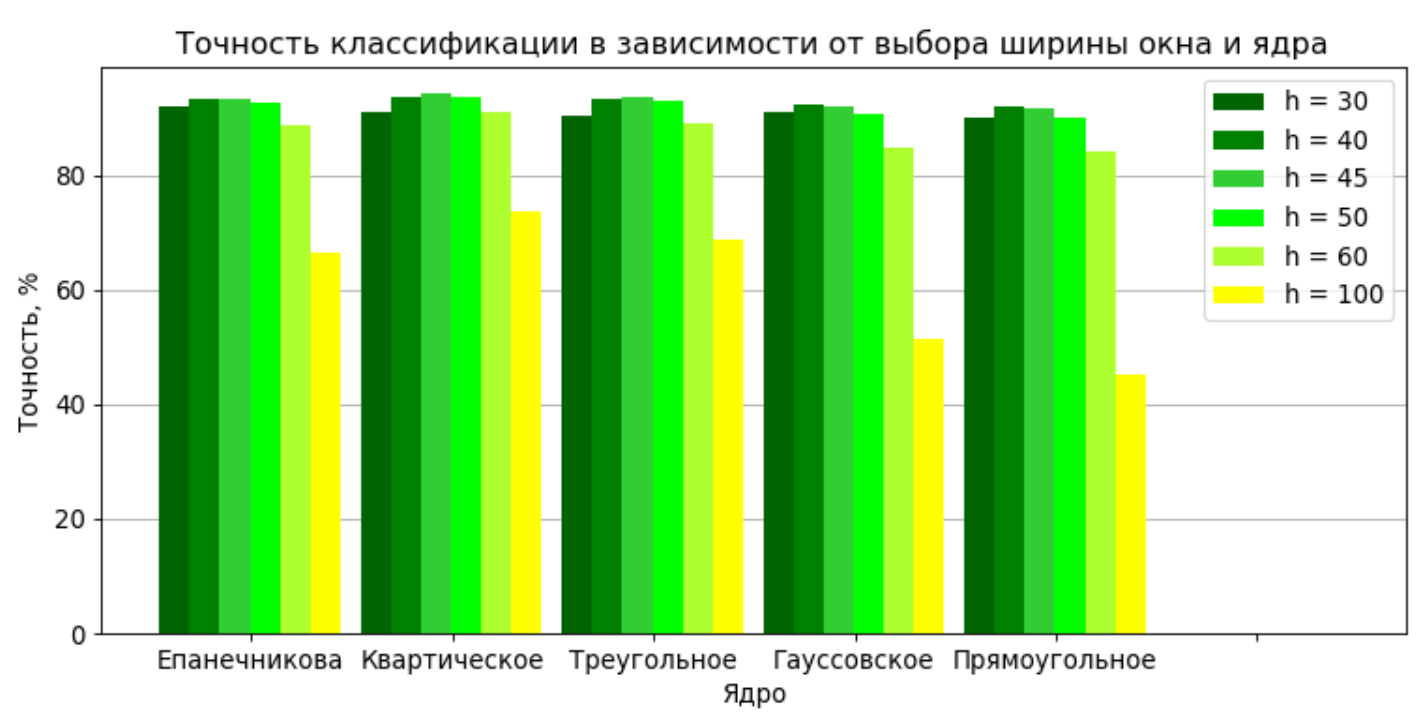

Рис. 6. Зависимость точности классификации методом Парзеновского окна от ширины окна и вида ядра

Достигнута точность 94,14 \% при ширине окна $h=45$, в качестве ядра было выбрано квадртическое ядро.

Метод опорных векторов с радиальным ядром функции (RBF)

Данный классификатор зависит от двух параметров - $C$ и $\gamma$.

Результаты исследования зависимости точности классификации от параметров $C$ и $\gamma$ представлены на рисунке 7. 


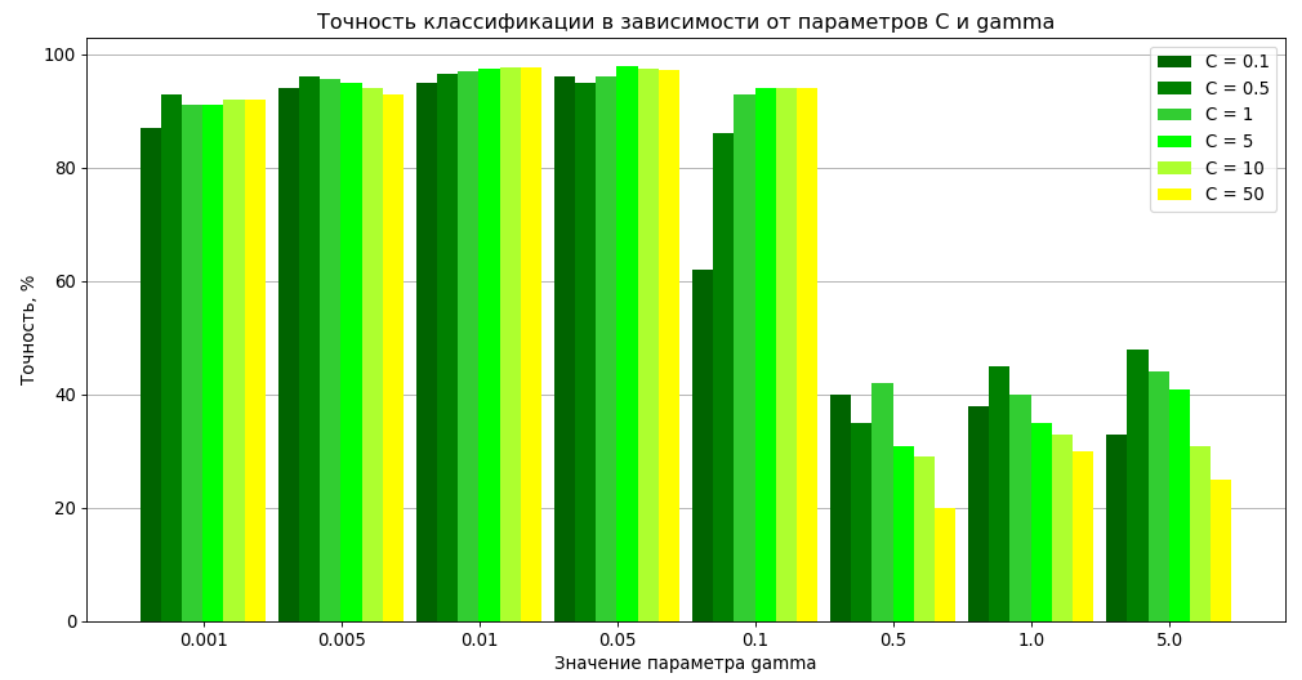

Рис. 7. График зависимости точности классификации методом опорных векторов от выбранных параметров $C$ и $\gamma$

Достигнута точность 98,52 \% при значении параметров $C=5$ и $\gamma=0.05$.

Подведем итоги исследования методов классификации. Результаты, полученные после подбора наилучших параметров для выборки MNIST, приведены в таблице 3.

Таблица 3

Исследование методов классификации

\begin{tabular}{|l|c|c|c|c|}
\hline \multicolumn{1}{|c|}{$\begin{array}{c}\text { Используемый } \\
\text { метод }\end{array}$} & $\begin{array}{c}\text { Качество клас- } \\
\text { сификации (доля } \\
\text { тестовой выбор- } \\
\text { ки равна 10 \%), } \\
\text { \% }\end{array}$ & $\begin{array}{c}\text { Время } \\
\text { классификации } \\
\mathbf{1 0 ~ 0 0 0 ~ о б ъ е к т о в , ~} \\
\text { секунды }\end{array}$ & $\begin{array}{c}\text { Время } \\
\text { обучения, } \\
\text { секунды }\end{array}$ & $\begin{array}{c}\text { Переобучение } \\
\text { классификатора } \\
\text { для каждого } \\
\text { символа }\end{array}$ \\
\hline $\begin{array}{l}\text { Мультиномиальная } \\
\text { логистическая ре- } \\
\text { грессия }\end{array}$ & 92.54 & 0.04 & 110 & Нет \\
\hline $\begin{array}{l}\text { Наивный Байесов- } \\
\text { ский классификатор }\end{array}$ & 92.44 & 0.02 & 0,6 & Нет \\
\hline $\begin{array}{l}\text { Метод } k \text { ближайших } \\
\text { соседей }\end{array}$ & 97.15 & 10561 & 110 & Да \\
\hline $\begin{array}{l}\text { Метод Парзеновско- } \\
\text { го окна }\end{array}$ & 94.14 & 20082 & 110 & Да \\
\hline $\begin{array}{l}\text { Метод опорных век- } \\
\text { торов с радиальным } \\
\text { ядром функции } \\
\text { (RВF) }\end{array}$ & 98.52 & 256 & 5426 & Нет \\
\hline
\end{tabular}

Таким образом, наилучшее качество классификации на выборке MNIST показали методы $k$ ближайших соседей и опорных векторов.

Метод $k$ ближайших соседей требует хранения всей выборки, размер которой приблизительно равен 80 Мб, а также для каждого нового классифицируемого объекта требуется переобучение классификатора, что сказывается на скорости работы приложения. Для одного символа классификация занимает примерно 1 секунду, то есть время классификации выражения в 5 символов составит примерно 5 секунд.

Метод опорных векторов осуществляет классификацию символа приблизительно за 0,026 секунды и не требует хранения данных, только значение полученных параметров при обучении. Данный подход обладает одним недостатком: обучение длится около полутора часов. Однако обучение требуется только один раз, поэтому после выполнения обучения на сервере пользователи смогут получить результат распознавания выражения в 10 символов приблизительно за 0,2 секунды. 


\section{Заключение}

Для решения задачи создания калькулятора с рукописным вводом в качестве классификатора выбран метод опорных векторов, так как при сравнительном анализе с другими методами он классифицировал объекты БД MNIST с максимальной среди исследованных методов точностью $(98,52$ \%) и высокой скоростью классификации (приблизительно 10 символов за 0,2 секунды).

Решение задачи интерпретации полученного математического выражения решается достаточно просто - использование встроенной функции eval() в языке Python в случае вычислений на сервере или аналогичной функции в языке JavaScript в случае выполнения в браузере.

В первоначальной версии приложения предусмотрено распознавание строковых выражений без возведения в степень, извлечения корней, дробей и т.п. При дальнейшем развитии приложения можно добавить больше математических возможностей.

\section{Лuтература}

1. MyScript Calculator. 2015. URL: https://play.google.com/store/apps/details?id=com.visionobjects. calculator\&hl=ru (дата обращения: 10.12.2017).

2. Touch Calculator. 2015. URL: https://github.com/zhaorz/Touch-Calculator (дата обращения: 10.12.2017).

3. Mathematical Expression Recognition. 2018. URL: http://cat.prhlt.upv.es/mer/ (дата обращения: 25.12.2017).

4. Hosmer D.W., Lemeshow S. Applied Logistic Regression. Wiley-InterScience Publ., 2000, 375 p.

5. Флах П. Машинное обучение. Наука и искусство построения алгоритмов, которые извлекают знания из данных; [пер. с англ. А.А. Слинкина]. М.: ДМК Пресс, 2015. 402 с.

6. Мерков А. Распознавание образов. Введение в методы статистического обучения. М.: Едиториал УPCC, 2011.256 c.

7. Коэльо Л.П., Ричарт В. Построение систем машинного обучения на языке Рython; [пер. с англ. А.А. Слинкина]. М.: ДМК Пресс, 2016. 302 с.

8. The MNIST database. URL: http://yann.lecun.com/exdb/mnist/ (дата обращения: 28.12.2017)

9. Вьюгин В.В. Математические основы теории машинного обучения и прогнозирования. М.: Изд-во МЦНМО, 2013. 390 c. 